\title{
FINITE ELEMENTS STRENGHT ANALYSIS OF A COMPRESSOR CONSISTING OF FOUR HELICAL ROTORS
}

\author{
Aleksander Nieoczym', Karol Szklarek ${ }^{1}$ \\ 1 Lublin University of Technology, Faculty of Mechanical Engineering, Nadbystrzycka Str. 36, 20-618 Lublin, \\ Poland, e-mail: a.nieoczym@pollub.pl, k.szklarek@pollub.pl
}

Received: 2017.05.15

Accepted: 2018.02.01

Published: 2018.03.01

\begin{abstract}
The present article reports the results of tests of a compressor consisting of four helical rotors. Finite element strength analysis of rotors with varied screw thread pitch was performed. The analysis allowed us to determine the stress-strain state of the rotor material and deformation of rotors as an effect of loads generated during operation of the compressor. The analysis also made it possible to calculate the frequencies of the free vibrations of the rotors.
\end{abstract}

Keywords: modal analysis, finite elements analysis, rotary-screw, compressor

\section{INTRODUCTION}

Rotary-screw compressors are positive-displacement machines in which screw-shaped rotors rotate around their own axis. They are manufactured either as twin-screw or multiple-screw devices. Compared with centrifugal compressors, rotary-screw compressors can achieve high compression pressures and high flow rates while retaining a small overall size. A limitation to the use of rotary-screw compressors is the strong heating up and vibrations of working parts. These disadvantageous features become more prominent along with increasing rotational speed of the moving elements and increasing compression pressure. Experimental research on rotary compressors is focused on two areas. A first area concerns creating mathematical models of compressor operation and validating their adequacy using physical models. A second area is the analysis of thermodynamic processes and the impact of the compressed medium on the functionality of the compressor. [3, 4, 6]

Discrepancies between the values of operating parameters of a compressor with three parallel helical rotors obtained from a mathematical model and corresponding data obtained from a test bench are described in [5]. The differences in the data were related to the value of the decrease in compression pressure and the value of the temperature to which the rotors heated up. The mathematical model was presented and the structural causes of the differences in the results were discussed. The sources of rotor vibration were identified and the possibilities of their elimination were addressed.

In $[7,10]$ the results of measurements of the frequency and intensity of vibrations in multiplerotor compressors were described. Analysis of vibration signals was performed using the Hilbert-Huang Transform (HHT) and the Empirical Mode Decomposition (EMD) models. Because interpretation of signals did not yield conclusive results, a method combining HHT with fourth order spectral analysis (the Kurtogram) was used. The possibility of using signals in the vibroacoustic diagnosis of compressors was highlighted. The results of tests of the effectiveness of artificial neural networks in the classification of the technical condition of the compressor or in predicting its condition were discussed.

The application of multi-body dynamic models for modelling and calculating the dynamic loads of twin-screw compressor bearings is dis- 
cussed in [9]. It has been shown that these loads are the result of the summation of two physical phenomena: cyclical changes in load pressure on the working surfaces of the rotors and rotor vibrations $[1,9]$.

These investigations are complemented by studies of numerical models. [8] describes the results of thermal deformation tests of screw rotors and their impact on the volumetric efficiency of a multiphase twin-screw compressor (GVFs). The calculations were performed using ANSYS software. The main variables were the flow rate and peripheral clearances.

[2] presents a semi-empirical procedure of modeling the capacity of a twin-screw compressor. Based on solid models and FEM simulations, the process of compression was divided into stages defined by changes in pressure due to leaks and heat exchange between the rotor and compressor chamber. Eight basic parameters of the model from which to calculate the effective flow rate, power on the output shaft, volumetric efficiency and heat loss were identified. This model did not take into account the presence of a lubricant.

\section{THE PRINCIPLE OF OPERATION OF THE COMPRESSOR}

The test compressor has four parallel helical rotors 1 (Fig. 1) with an oval crosssection. The rotors terminate on both sides in flat faces $P c$. Along these flat faces, the rotors adjoin the flat surface $P o$ of chamber 2 with centrally arranged holes $O t$ which form flow channels for the working medium.

Each of the rotors can be divided into a positive displacement section $S p$ (Fig. 2) and two sealing sections $S k$ with flat faces $P c$. In the displacement section $S p$, the cross-section of the side surface of the rotor does not change along its axis.

The task of the displacement sections of the rotors is to displace the working medium and push it toward the discharge port. The sealing sections are designed to permanently separate the working space from the external space surrounding the meshing rotors. The compressor operates at two cycles per revolution of the rotor. Its momentary volume flow rate changes in a cyclic manner, and these changes are due to fluctuations in volume growth in the part of the working space between the rotors which opens toward the discharge port. Changes in the volume of the sealed working space of the compressor at different stages of screw rotation are shown in Fig. 3.

\section{PRELIMINARY ASSUMPTIONS AND EXPERIMENTAL DESIGN}

The numerical analysis involved:

- determination of reduced stresses and displacements in three selected angular positions of the rotor at three different rotational speeds and

- determination of the first three frequencies of free vibrations of the rotor (modal analysis) in order to identify the mode of these vibrations.

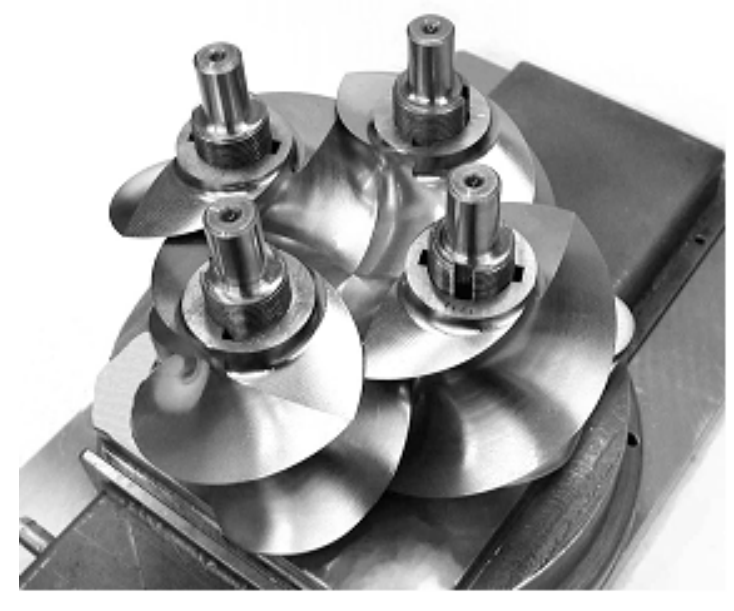

a

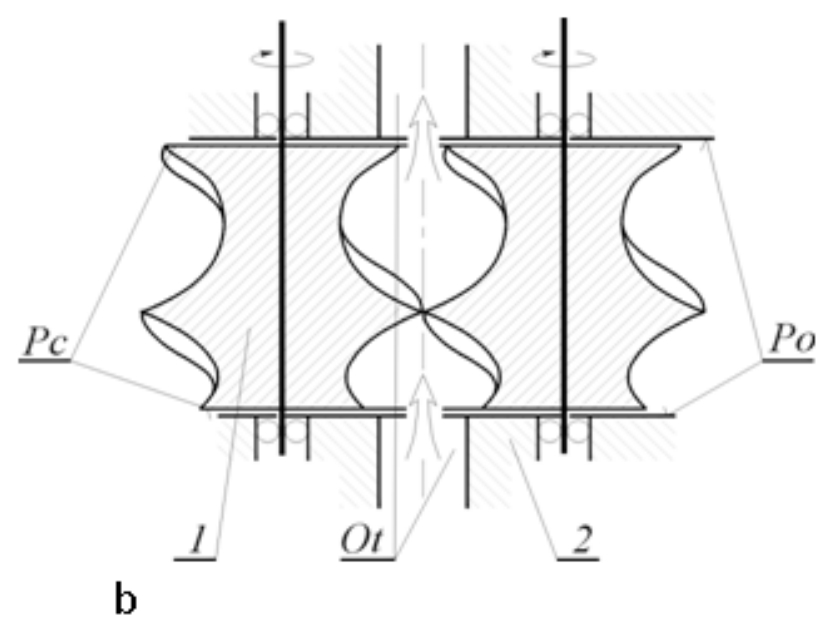

b

Fig. 1. Compressor with four parallel helical rotors: a- prototype (removed cover) , b- axial cross - section: 1- rotor, 2- cover, $\mathrm{P}_{\mathrm{c}}$ - flat faces, $\mathrm{P}_{\mathrm{o}}$ - flat surface of cover, $\mathrm{Ot}$ - hole 


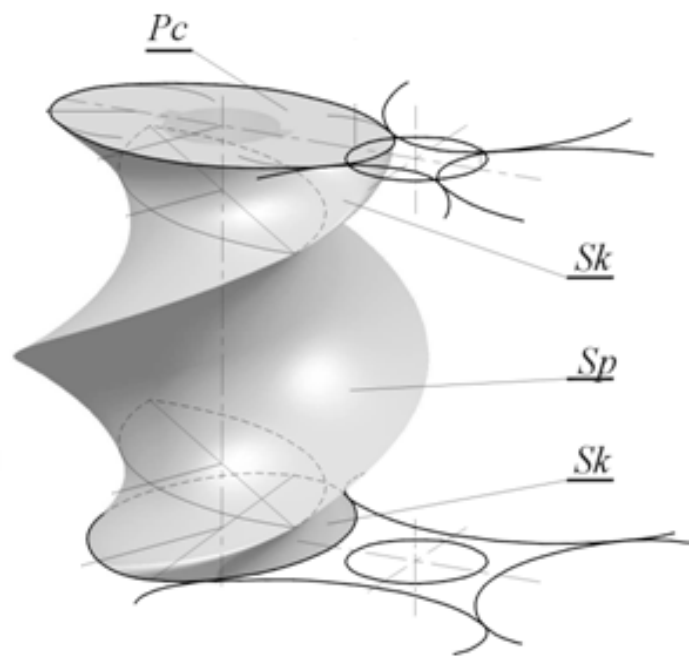

Fig. 2. Helical rotors [11]: $\mathrm{Sp}$ - displacement section, $\mathrm{S}_{\mathrm{k}}-$ sealing sections, $\mathrm{Pc}-$ flat faces

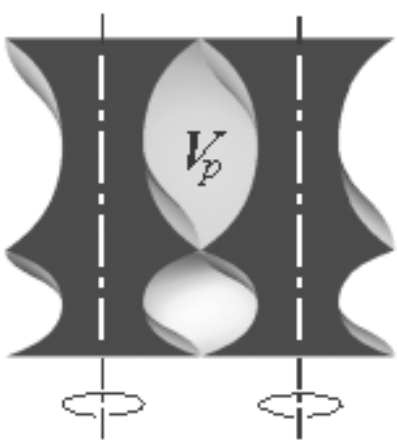

a

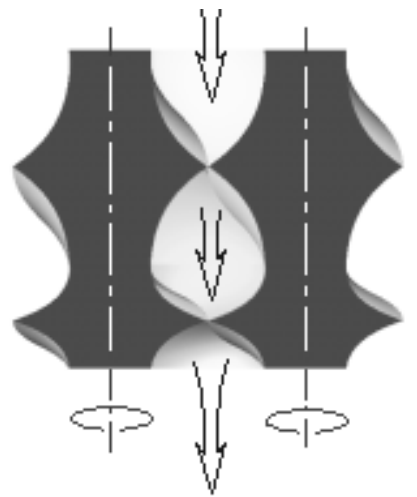

b

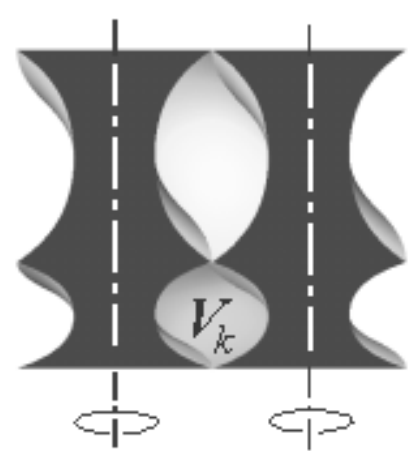

C

Fig. 3. Changes in the volume of the sealed working space of the compressor: a- after closure of the work area from the inlet port, b- in an intermediate position, c- before opening the working space towards the discharge port

Calculations were carried out for three rotational speeds: $\mathrm{n}=2000 ; 4000 ; 6000$ [rev/min].

The following angular positions (extreme positions) of the rotor were considered:

- WT0 - angular position of $0^{\circ}$

- WT60 -angular position of $60^{\circ}$

- WT120 -angular position of $120^{\circ}$

Figure 4 shows the meshing screws at the three selected angular positions along with a division into pressure zones.

Angular position WT0 was defined as the position of the rotor in which the volume of air subjected to compression was sealed between the rotors. The air in the working space and at the intake port was at atmospheric pressure (marked in yellow). At the discharge port (red color) the pressure of the compressed medium was $0.6 \mathrm{MPa}$. The color blue marks high pressure zones between the rotor surfaces and the cham- ber. Because the rotors operated in a sealed and airtight chamber, it was assumed that the pressure between the rotors and the chamber would stabilize as an effect of clearances. For calculation purposes, it was assumed that the pressure of compressed medium in the chamber was 0.14 $\mathrm{MPa}$. Position WT60 was defined as the moment just before maximum compression of air to 0.94 $\mathrm{MPa}$ (marked in orange) and opening of the sealed working space. The last position tested, WT120, was the moment in which the working chamber is opened and the air is pushed into the discharge port of the compressor.

Preliminary bench tests were performed at the maximum compression pressure of the working medium of $0.94 \mathrm{MPa}$. The goal of the tests was to determine whether the meshing rotors provided an appropriate working space and to establish the impact of the formation of variable thermal fields 


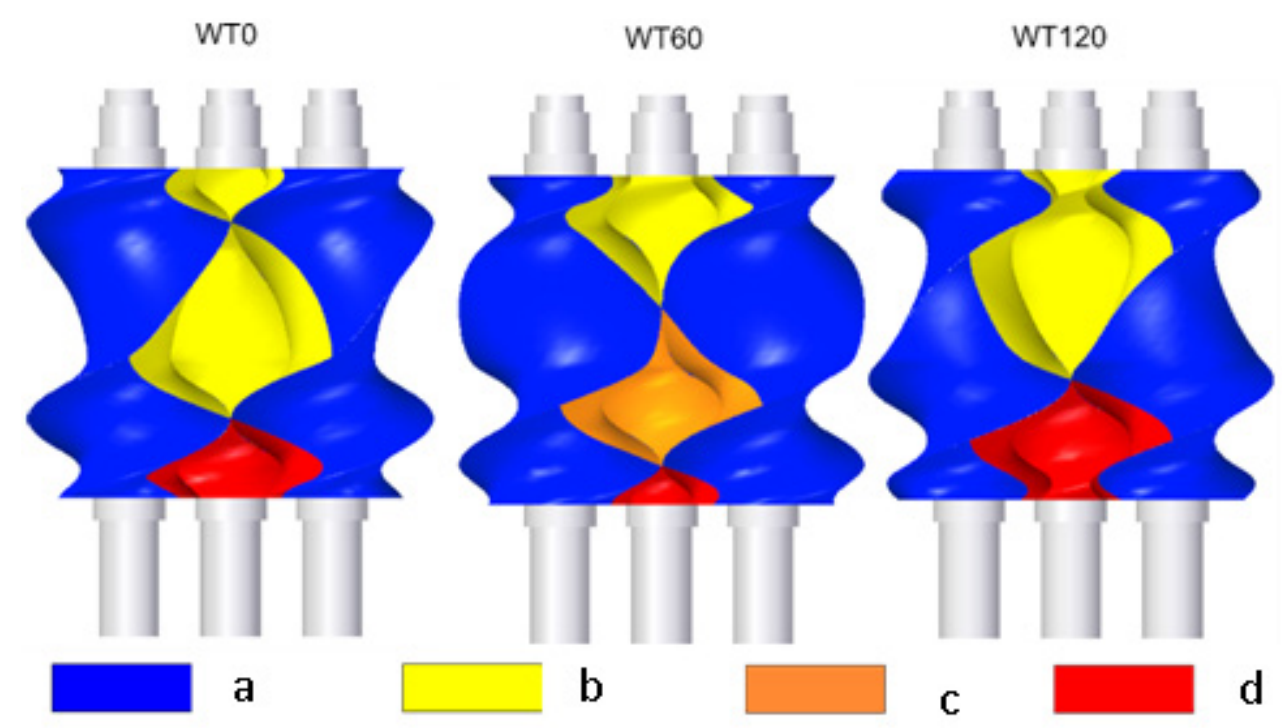

Fig. 4. Pressure zones at selected angular positions: a -pressure of compressed medium in the chamber, $\mathrm{b}$ - atmospheric pressure, $\mathrm{c}$ - compression pressure, $\mathrm{d}$ - high pressure

on the efficiency of the compressor. Additionally, the values of free vibration frequencies and probability of excitation of resonant vibrations were investigated.

\section{RESULTS OF FE ANALYSIS}

At the stage of preparation of computational models, a 3D model of the rotor was subjected to modifications which concerned the division of the rotor's working surfaces. The division was introduced to identify areas in which the pressure acting on the surfaces of the rotor depended on the angle of rotation of the rotor (Fig. 5). The designated areas were used to assign pressure load conditions.

The models were discretized using a tetragonal finite element mesh consisting of 10-node second-order finite elements C3D10.

Conclusions from the numerical analysis:

- Reduced stresses on the working surfaces of the rotor did not exceed $4 \mathrm{MPa}$ (Table 1). The highest values of reduced stresses were obtained in places where the cross-section of the rotor changed (rotor grading) and were associated with the notch effect (Fig. 6). Figure 7 compares the maximum values of reduced stresses depending on the rotational speed of the rotor at the location of the notch.

- The maximum total displacement for all load states did not exceed $2 \mu \mathrm{m}$ when technological clearance was $0.07 \mathrm{~mm}$ [1.3]. Increasing of the rotational speed in each of the investigated an- gular positions of the rotor caused an increase in the value of maximum resultant displacements. The analysis showed that the location of maximum displacements depended on the angular position of the rotor (Fig 8). This was directly related to changes in the load (pressure) acting on the rotor. The largest maximum displacements were observed for the angular position of $120^{\circ}$ (WT120).

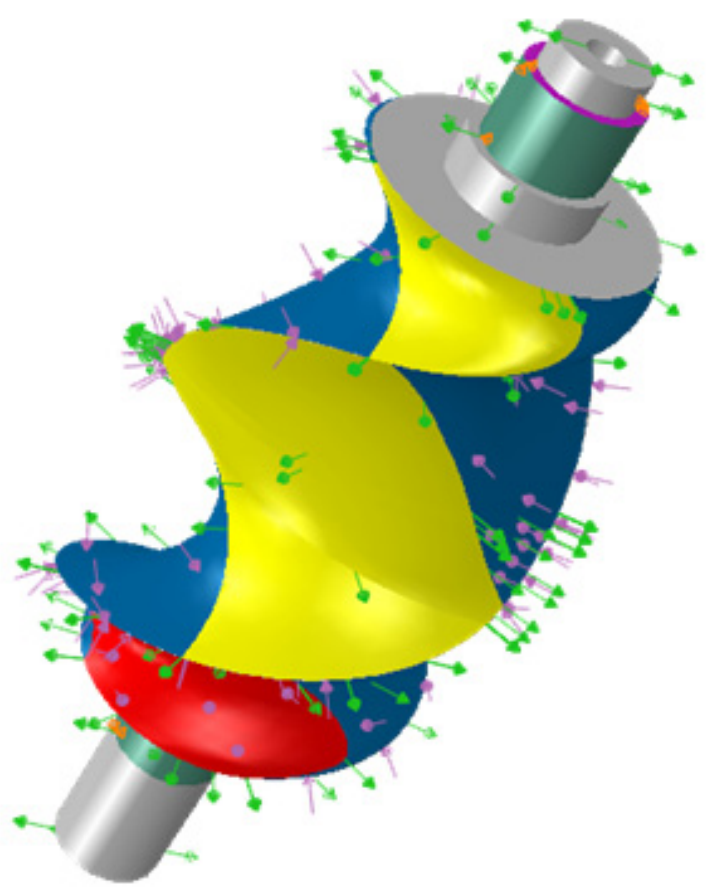

Fig. 5. Load distribution and division of the rotor's working surfaces 
Table 1. Sample results of FE analysis

\begin{tabular}{|c|c|}
\hline 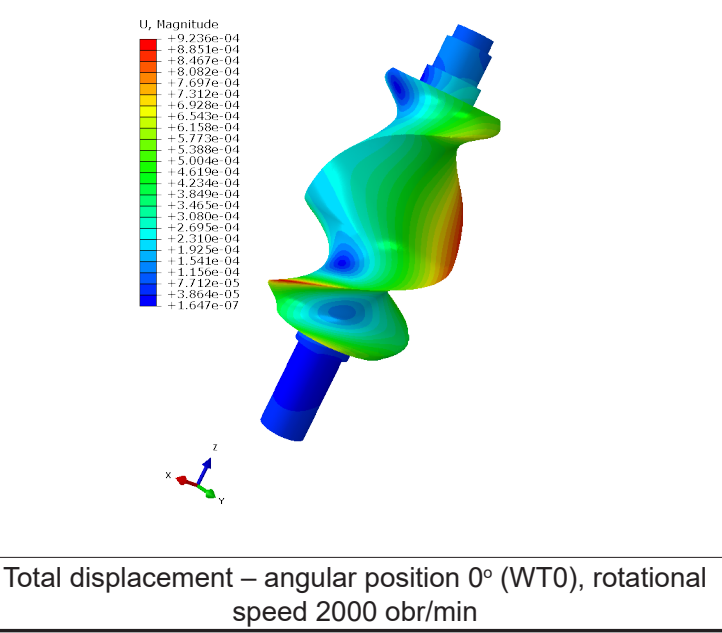 & 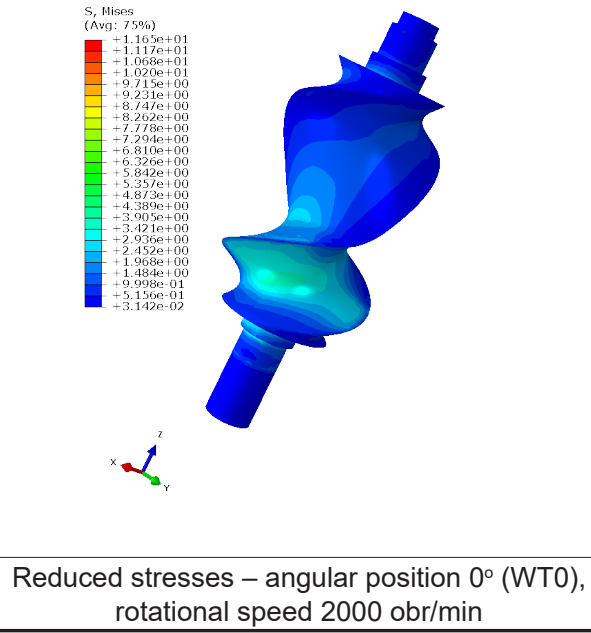 \\
\hline Total displacement - angular position $0^{\circ}$ (WT0), rotational & 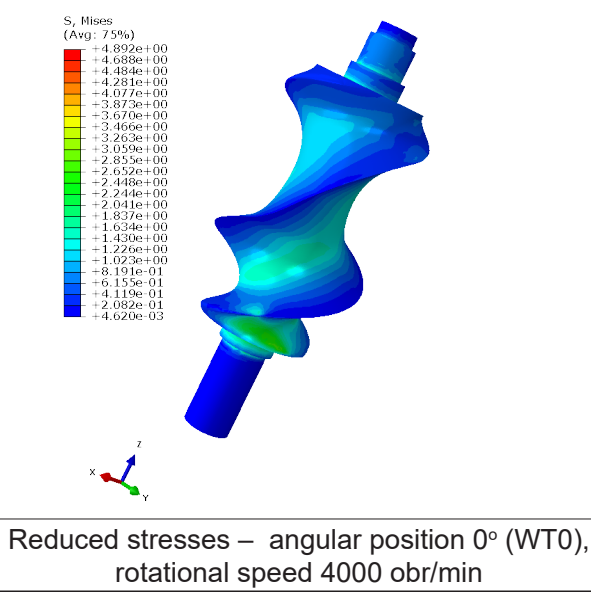 \\
\hline $\begin{array}{c}\text { Total displacement - angular position } 0^{\circ} \text { (WT0), rotational } \\
\text { speed } 6000 \mathrm{obr} / \mathrm{min}\end{array}$ & 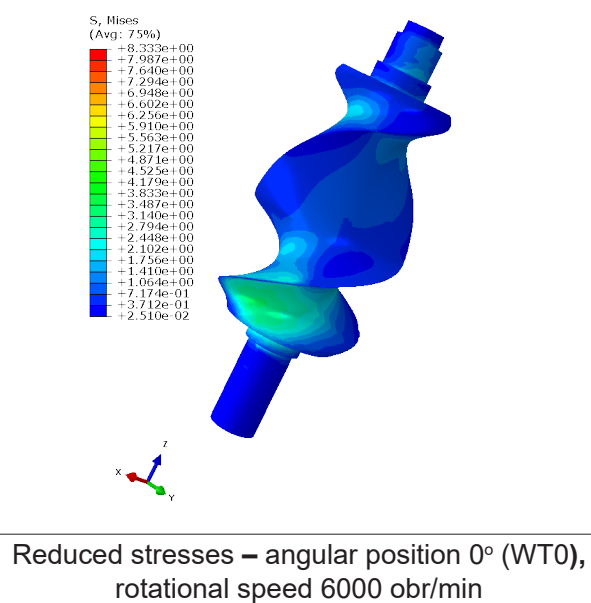 \\
\hline
\end{tabular}

Additionally, axial and radial displacements of the rotor were analyzed (Fig. 9-11). The maximum values of these displacements were recorded for position WT120 at a rotational speed of $6000 \mathrm{rev} / \mathrm{min}$.

Axial deformation was the largest on the edges of the rotor in the sealing section. These areas are located in the vicinity of the discharge port where there compression pressure reaches maximum values.

The results showed that in the portion of the rotor with a large screw thread pitch (lead), the largest effect on deformations was exerted by radial displacement, while in parts of the rotor with small lead, deformations were related to axial displacement. 


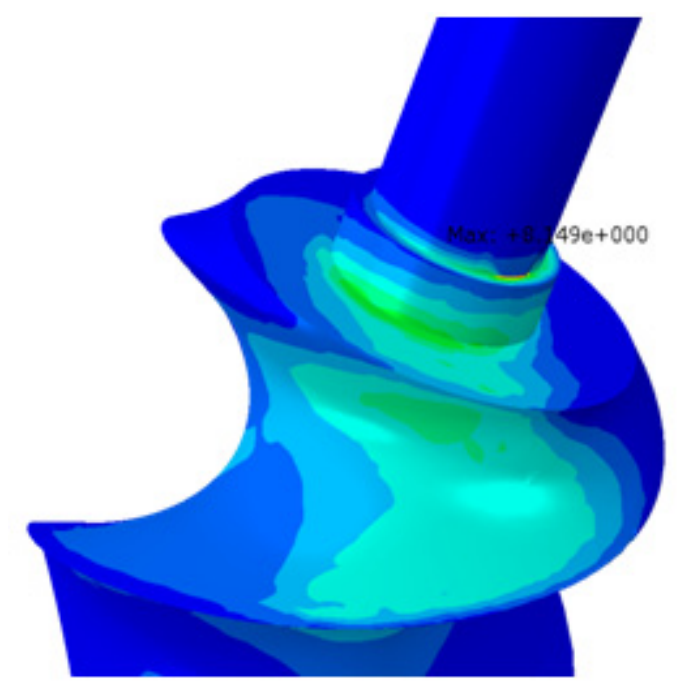

Fig. 6. The places of highest values of reduced stresses

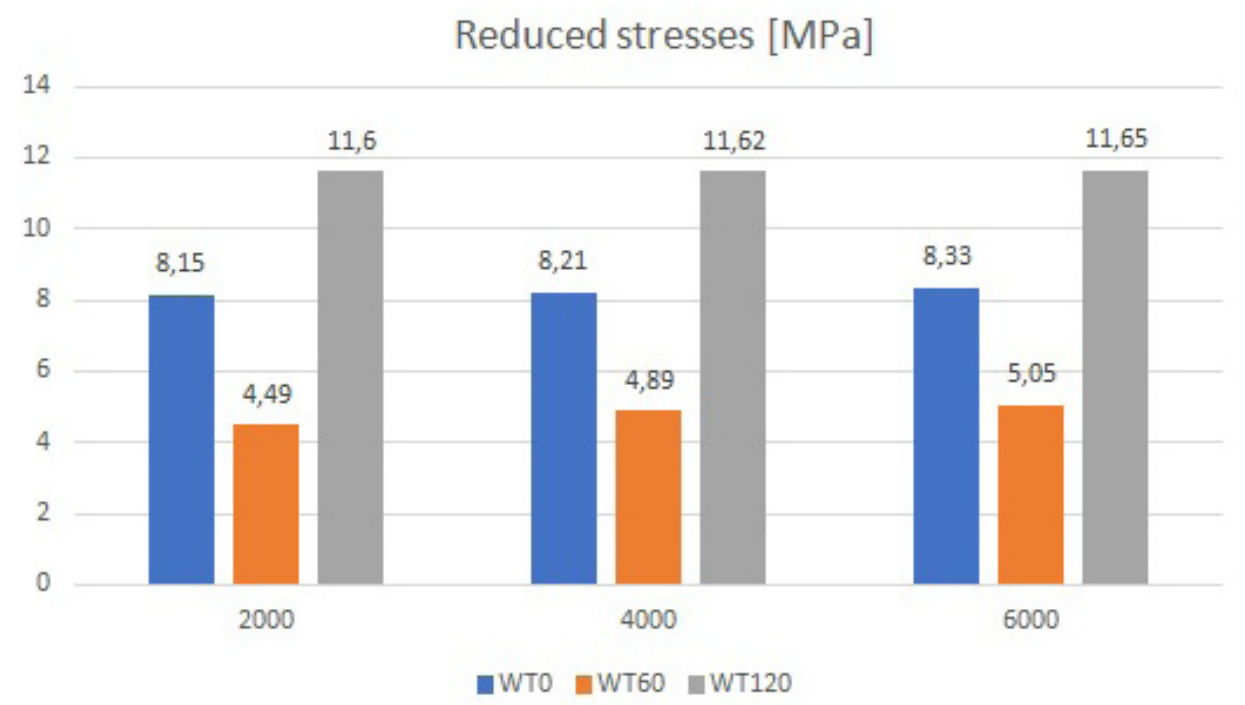

Fig. 7. Maximum values of reduced stresses at the location of the notch.

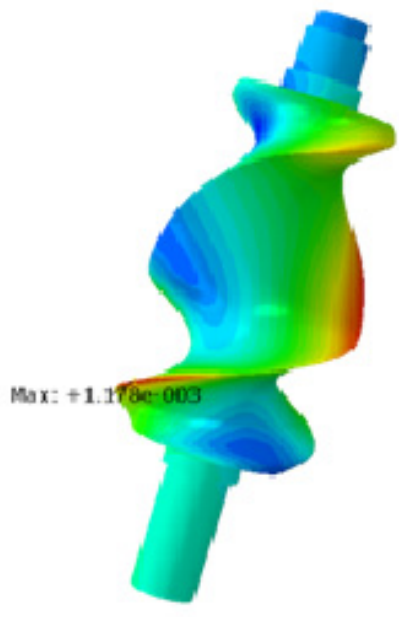

a

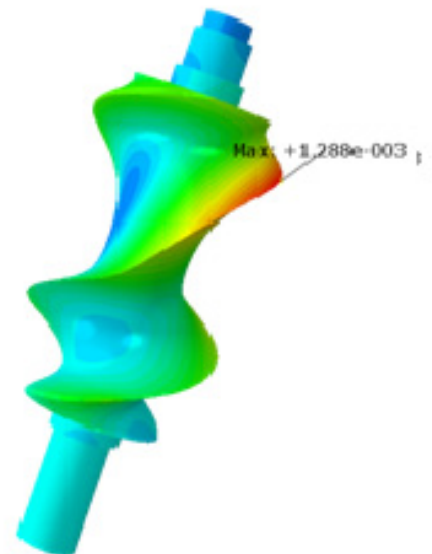

b

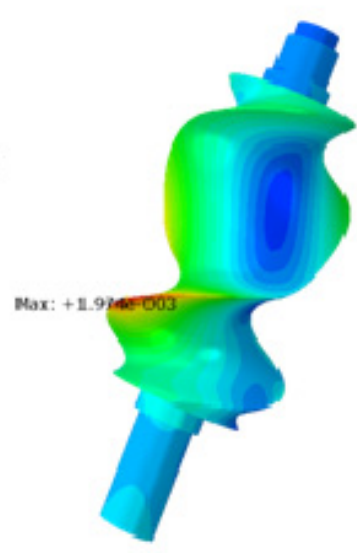

C

Fig. 8. Location of maximum accidental displacements: a - WT0 , b - WT60, c-WT120 


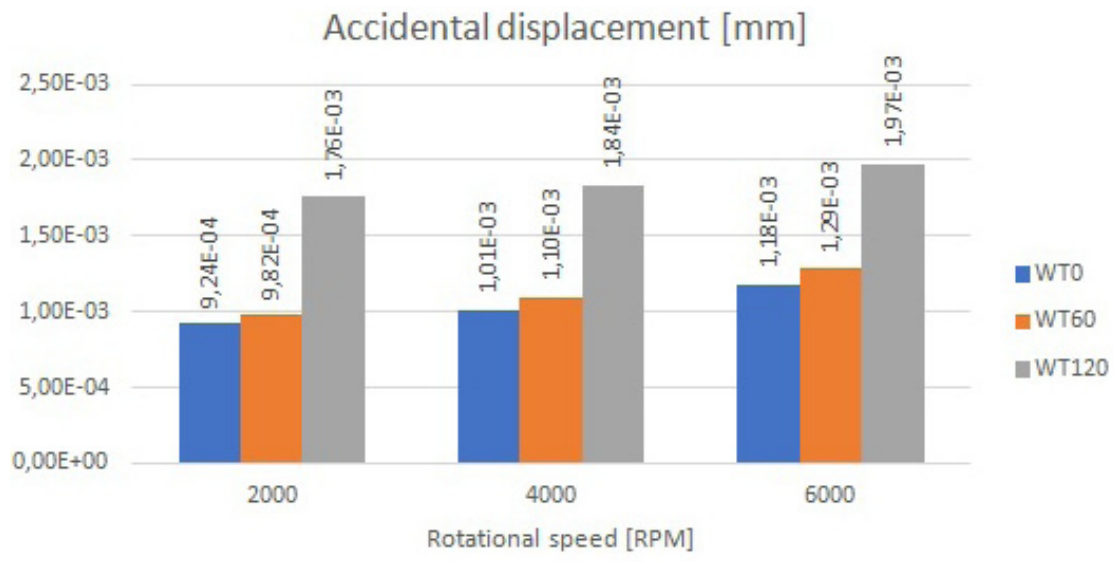

Fig. 9. Maximum values of accidental displacement

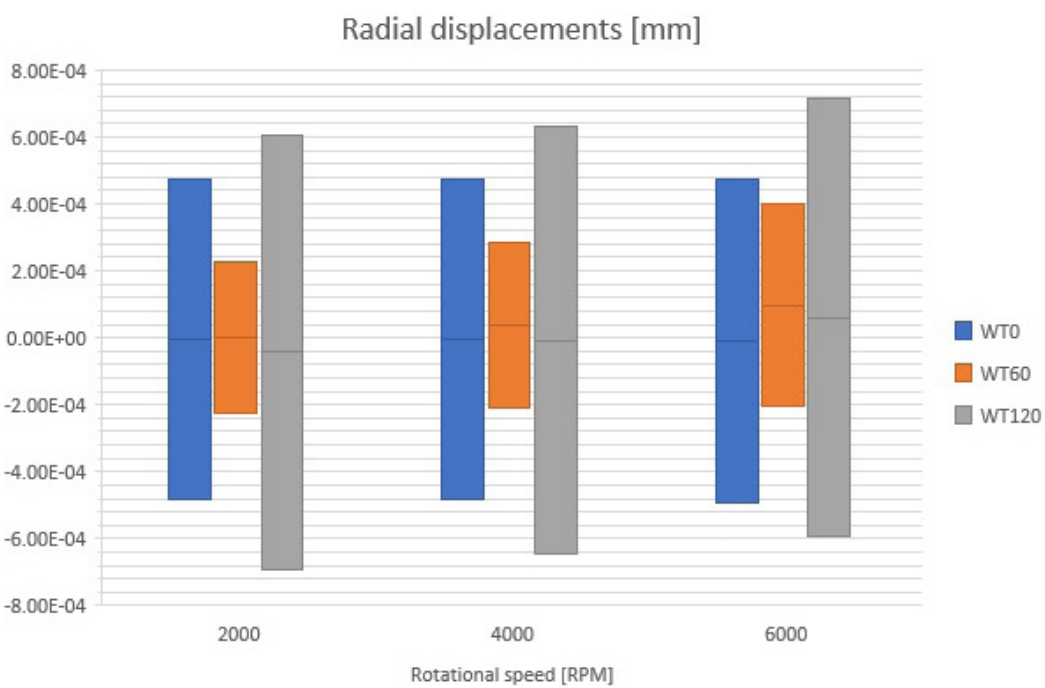

Fig. 10. Radial displacements for three selected angular positions

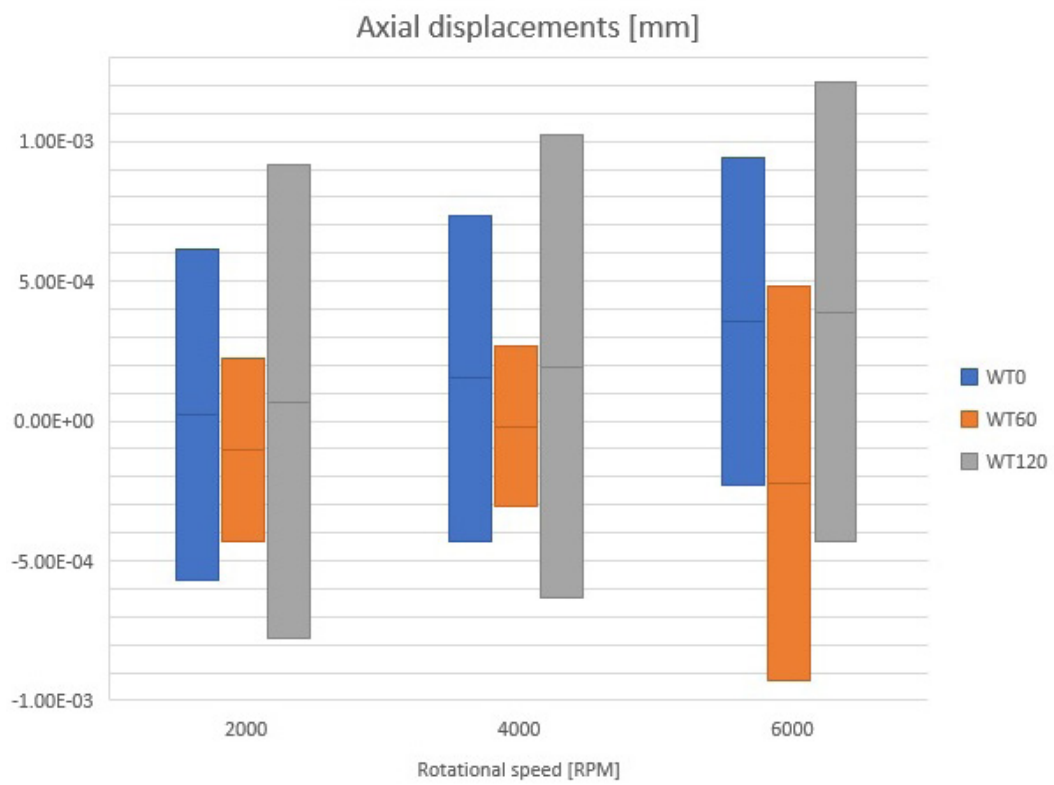

Fig. 11. Axial displacements for three selected angular positions 


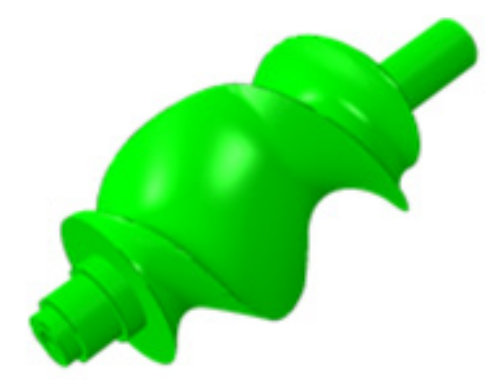

a

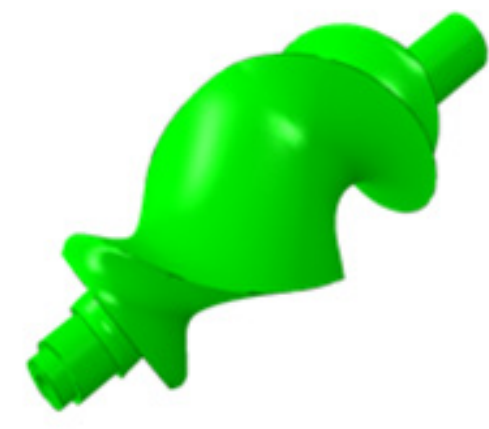

b

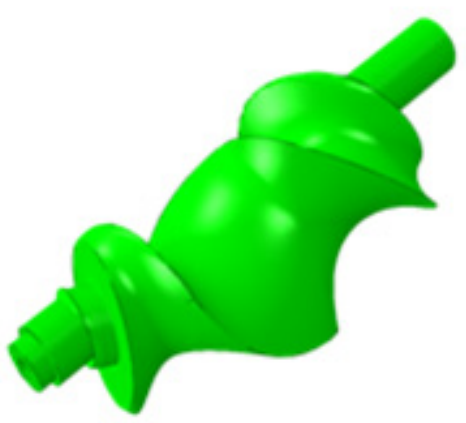

C

Fig. 12. Results of numerical modeling of free-vibration frequency of the rotor: a- mode $n 1-2729$ [Hz], $\mathrm{b}-$ mode n2 - $3477[\mathrm{~Hz}]$, c- mode n3 - $3827[\mathrm{~Hz}]$

The modal analysis demonstrated that operation of the rotor was not affected by resonant states induced by rotational speed and loadings related to air compression. The first free-vibration frequency of the rotor was $2729 \mathrm{~Hz}$, which was well above the expected range of the rotor's rotational speed $(100 \mathrm{~Hz})$ and the twice-as-high frequency of the loading equal to the number of compressor cycles $(200 \mathrm{~Hz})$. Changing of load conditions (rotational speed and angular position of the rotor) did not affect the frequency of the rotor's free vibrations. The second and third modes were flexural modes. In the first vibration mode, deformations were observed in the form of torsion and radial and axial displacements - Fig. 12.

\section{CONCLUSIONS}

Numerical analysis allowed us to determine the levels of reduced stresses, deformations, and frequency of free vibrations of the rotor. The maximum values of reduced stresses were low and did not pose a risk to the structure of the rotor. Frequency analysis showed that the structure of the compressor was not threaten by resonant states, either. The obtained deformation values indicated that the deformations should not affect the clearance between rotor surfaces and the chamber of the compressor.

\section{REFERENCES}

1. Fujiwara A., Matsuo, K., Yamashita, H. Vibration analysis of oil-injected twin-screw compressors using simple simulated waveforms.. Proceedings of the Institution of Mechanical Engineers, 225, 105-116. 2011

2. Giuffrida A.; A semi-empirical method for assess- ing the performance of an open-drive screw refrigeration compressor. Applied Thermal Engineering 83, 813-823, 2016

3. Hsieh, S.H., Shih, Y.C., Hsieh,W.H., Lin, F.Y., Tsai, M.J., Performance analysis of screw compressors - numerical simulation and experimental verification. Proceedings of the Institution of Mechanical Engineers. 226, 968-980. 2012

4. Mucchi E., Dalpiza G.: Experiment validation of a model for the dynamic analysis of gear pumps. Proceedings of the ASME 2013 International Design Engineering Technical Conferencesand Computersand Informationin Engineering Conference, Portland, 1-10, August 2013

5. Li W., Lu H., Zhang Y., Zhu C., Lu X.: Vibration analysis of three-screw pumps under pressure loads and rotor contact forces. Journal of Sound and Vibration, 360, 74-96, 2016,

6. Stošic N., Smith I.K., Kovacevic A., Mujic E.: Review of mathematical models in performance calculation of screw compressors, International Journal of Fluid Machinery and Systems 4 (2), 271-288 2011

7. Xiong X., Yang S., Gan C.: A new procedure for extracting fault feature of multi-frequency signal from rotating machinery. Mechanical Systems and Signal Processing 32, 306-319, 2012

8. Yin X. , Cao F., Pan S., Song Y., Fei J., Wang X.: Numerical investigation on screw rotor deformation and influence on volumetric efficiency of the twin-screw multiphase pump. Applied Thermal Engineering, 111, 1111-1118, 2017

9. Yu-Ren Wu, Van-The Tran: Dynamic response prediction of a twin-screw compressor with gasinduced cyclic loads based on multi-body Dynamics. International Journal of refrigeration, 65, 111-128, 2016.

10. Zhang Y.P., Huang S.H., Hou J.H.: Continuous wavelet grey moment approach for vibration analysis of rotating machinery, Mechanical Systems and Signal Processing, 20, 1202-1220, 2006.

11. Zniszczyński A.: Displacement machines with four parallel synchronous rotors. Mechanism and Machine Theory, 44, 1677-1688, 2009. 